\title{
A NOTE ON A THEOREM OF AHERN AND SARASON
}

\author{
JAMES DUDZIAK
}

\begin{abstract}
A strong weak* maximality theorem due to Ahern and Sarason is simply deduced from a more easily obtained and weaker maximality theorem of Garnett.
\end{abstract}

Throughout this note it is assumed that $K$ is a compact subset of the complex plane $\mathrm{C}$ such that $R(K)$ is hypodirichlet. Recall that this means that the uniform closure of $\operatorname{Re} R(K)$ in $C_{\mathbf{R}}(\partial K)$ has finite codimension and that the real-linear span of $\log \left|R(K)^{-1}\right|$ is uniformly dense in $C_{\mathbf{R}}(\partial K)$. For $z \in K$, it follows that $M_{z}$, the set of representing measures on $\partial K$ for the complex homomorphism $z$ of $R(K)$, is finite dimensional and that there is a unique positive measure $\lambda_{z}$ on $\partial K$ such that $\log |f(z)|=\int \log |f| d \lambda_{z}$ for each $f \in R(K)^{-1}$. This measure $\lambda_{z}$ is called the logmodular measure or Arens-Singer measure for the complex homomorphism $z$ of $R(K)$. Consideration of $\exp R(K)$ shows $\lambda_{z}$ to be in $M_{z}$. Denote the weak* closure of $R(K)$ in $L^{\infty}\left(\lambda_{z}\right)$ by $H^{\infty}\left(\lambda_{z}\right)$. Ahern and Sarason have proven the following.

THEOREM A. $H^{\infty}\left(\lambda_{z}\right)$ is a maximal weak ${ }^{*}$ closed subalgebra of $L^{\infty}\left(\lambda_{z}\right)[\mathrm{AS1}, 14.5$ and 15].

Notice that Theorem $\mathrm{A}$ is a generalization of the well-known fact that the algebra $H^{\infty}(d \theta / 2 \pi)$ of bounded analytic functions on the unit disc is a maximal weak* closed subalgebra of $L^{\infty}(d \theta / 2 \pi)$. In [AS2], Ahern and Sarason comment that Theorem A "comes near the end of a long paper and depends in an essential way on many of the earlier results in that paper. A more direct proof of Theorem A ... would ... be of interest." A little later Garnett obtained a short proof of the following weaker version of Theorem A.

THEOREM B. $H^{\infty}\left(\lambda_{z}\right)$ is maximal among the subalgebras of $L^{\infty}\left(\lambda_{z}\right)$ on which $\lambda_{z}$ is multiplicative [Gar, 3.7 and the comment afterward or $\mathbf{S}, 26.34$ ].

The purpose of this note is to show that with a little effort Theorem A can be deduced from Theorem B. The only other fact needed from the theory of hypodirichlet algebras is the following. It lies close to the surface.

THEOREM C. Each measure in $M_{z}$ is absolutely continuous with respect to $\lambda_{z}$ [AS1, Corollary 1 to 3.1 or $\mathbf{S}, 23.13$ and 26.32].

Received by the editors December 10, 1984.

1980 Mathematics Subject Classification (1985 Revision). Primary 30E10, 30H05; Secondary 46J10, $46 \mathrm{~J} 15$. 
A few comments on the references [Gar] and [S] seem called for. First, both references define $H^{\infty}\left(\lambda_{z}\right)$ differently than here. However, both references contain propositions showing that the two definitions lead to the same result, namely, [Gar, 3.6] and [S, 23.14]. Second, both references assume not that $R(K)$ is hypodirichlet, but more stingently that $\mathbf{C} \backslash K$ has only finitely many components. However, an inspection of the proofs of the results quoted reveal that all that is used is the hypodirichlicity of $R(K)$. Incidently, [S, 26.26] shows that $R(K)$ is hypodirichlet whenever $\mathbf{C} \backslash K$ has only finitely many components.

Define a complex homomorphism $\phi_{z}$ of $H^{\infty}\left(\lambda_{z}\right)$ by $\phi_{z}(f) \equiv \int f d \lambda_{z}$ for each $f \in H^{\infty}\left(\lambda_{z}\right)$. Then

$$
\phi_{z}(f)=f(z) \text { for each } f \in R(K) .
$$

Let $\mathscr{M}$ denote the maximal ideal space of $L^{\infty}\left(\lambda_{z}\right)$ and look upon $H^{\infty}\left(\lambda_{z}\right)$ as residing in $C(\mathscr{M})$. Although $H^{\infty}\left(\lambda_{z}\right)$ need not be a uniform algebra on $\mathscr{M}$ (it need not separate points), retaining uniform algebra terminology, a positive measure $\mu$ on $\mathscr{M}$ such that $\phi_{z}(f)=\int f d \mu$ for each $f \in H^{\infty}\left(\lambda_{z}\right)$ is called a representing measure for the complex homomorphism $\phi_{z}$ of $H^{\infty}\left(\lambda_{z}\right)$. Notice that $L^{1}\left(\lambda_{z}\right) \subseteq L^{1}\left(\lambda_{z}\right)^{* *}=$ $L^{\infty}\left(\lambda_{z}\right)^{*}=C(\mathscr{M})^{*}=$ the space of measures on $\mathscr{M}$. The only nonstandard fact needed from the general theory of uniform algebras is the following, valid for any $R(K)$, hypodirichlet or not. It too lies close to the surface.

THEOREM D. The set of representing measures on $\mathscr{M}$ for the complex homomorphism $\phi_{z}$ of $H^{\infty}\left(\lambda_{z}\right)$ is the weak* closure in the space of measures on $\mathscr{M}$ of $M_{z} \cap L^{1}\left(\lambda_{z}\right)$ [Gam, IV.2.3].

Proof of Theorem A. Let $A$ be a weak* closed subalgebra of $L^{\infty}\left(\lambda_{z}\right)$ containing $H^{\infty}\left(\lambda_{z}\right)$. It must be shown that $A=L^{\infty}\left(\lambda_{z}\right)$ or $H^{\infty}\left(\lambda_{z}\right)$. If $z \in \partial K$, then $\lambda_{z}$ is clearly the point mass at $z$ and the desired conclusion follows trivially. Because of this, it may, and will, be assumed that $z \in K^{0}$.

Case I $\left((\zeta-w)^{-1} \in A\right.$ for each $w$ in the part of $R(K)$ containing $\left.z\right)$. Let $\mu$ be a weak* continuous annihilator of $A$, i.e., let $\mu$, absolutely continuous with respect to $\lambda_{z}$, be such that $\int f d \mu=0$ for each $f \in A$. Since $R(K) \subseteq A, \mu \perp R(K)$. Assume that $\hat{\mu}(w) \neq 0$. By the proof of [Gam, II.8.5], $\mu(\zeta) /(\hat{\mu}(w)(\zeta-w))$ is a complex representing measure for the complex homomorphism $w$ of $R(K)$. By [Gam, II.2.2], there exists a representing measure for the complex homomorphism $w$ of $R(K)$ that is absolutely continuous with respect to $\mu$. Since $\mu$ is absolutely continuous with respect to $\lambda_{z}$, [Gam, VI.2.2] implies that $w$ is in the part of $R(K)$ containing $z$. But then $(\zeta-w)^{-1} \in A$ by assumption, so $\hat{\mu}(w)=\int d \mu(\zeta) /(\zeta-w)=0$. This contradiction shows that $\hat{\mu}=0$ area-a.e. on C. By [Gam, II.8.3], $\mu=0$. By the Hahn-Banach theorem, $A=L^{\infty}\left(\lambda_{z}\right)$.

Case II. $\left((\zeta-w)^{-1} \notin A\right.$ for some $w$ in the part of $R(K)$ containing $\left.z\right)$. Theorem $\mathrm{C}$ and [Gam, VI.1.2] imply that $\lambda_{w}$ and $\lambda_{z}$ are mutually absolutely continuous. Hence $L^{\infty}\left(\lambda_{w}\right)=L^{\infty}\left(\lambda_{z}\right)$ and $H^{\infty}\left(\lambda_{w}\right)=H^{\infty}\left(\lambda_{z}\right)$. Because of this, it may, and will, be assumed that $w=z$. Then, since $(\zeta-z)^{-1} \notin A$, there exists a complex homomorphism $\phi$ of $A$ such that $\phi(\zeta)=z$. 
Let $f \in H^{\infty}\left(\lambda_{z}\right)$. Choose a net $\left\{f_{\alpha}\right\}$ of rational functions with poles off $K$ converging weak* in $L^{\infty}\left(\lambda_{z}\right)$ to $f$. Set $g(\zeta) \equiv\left(f(\zeta)-\phi_{z}(f)\right) /(\zeta-z)$. Since $z \in K^{0}$ and $\lambda_{z}$ is on $\partial K$, one easily verifies that $\left(f_{\alpha}(\zeta)-f_{\alpha}(z)\right) /(\zeta-z)$ converges weak* in $L^{\infty}\left(\lambda_{z}\right)$ to $g$. Hence $f(\zeta)-\phi_{z}(f)=g(\zeta)(\zeta-z)$ where $g \in H^{\infty}\left(\lambda_{z}\right)$. Clearly then

$$
\phi(f)=\phi_{z}(f) \text { for each } f \in H^{\infty}\left(\lambda_{z}\right) .
$$

Define a linear functional $L$ on the real-linear span of $\log \left|A^{-1}\right|$ by $L\left(\sum_{n} c_{n} \log \left|f_{n}\right|\right)$ $\equiv \sum_{n} c_{n} \log \left|\phi\left(f_{n}\right)\right|$. A standard argument shows $L$ to be well defined with norm one (see, for example, the proof of [Gam, IV.7.1]). Invoke the Hahn-Banach theorem to get a norm-preserving linear extension of $L$ to all of $C_{\mathbf{R}}(\mathscr{M})$ and then invoke Riesz's representation theorem to get a measure $\mu$ on $\mathscr{M}$ of norm one such that

$$
\log |\phi(f)|=\int \log |f| d \mu \quad \text { for each } f \in A^{-1} .
$$

Consideration of $\exp A$ shows that

$$
\phi(f)=\int f d \mu \quad \text { for each } f \in A .
$$

Since $H^{\infty}\left(\lambda_{z}\right) \subseteq A,(* *)$ and $(* * *)$ imply that $\phi_{z}(f)=\int f d \mu$ for each $f \in H^{\infty}\left(\lambda_{z}\right)$. As $\int d \mu=1=\int d|\mu|, \mu$ is positive. Hence $\mu$ is a representing measure for the complex homomorphism $\phi_{z}$ of $H^{\infty}\left(\lambda_{z}\right)$. By Theorem $\mathrm{D}, \mu$ is in the weak* closure in the space of measures on $\mathscr{M}$ of $M_{z} \cap L^{1}\left(\lambda_{z}\right)$. As $M_{z} \cap L^{1}\left(\lambda_{z}\right)$ is finite dimensional by the hypodirichlicity of $R(K)$, it is also weak* closed. Thus $\mu$ is actually a positive measure on $\partial K$ absolutely continuous with respect to $\lambda_{z}$.

Since $R(K) \subseteq H^{\infty}\left(\lambda_{z}\right) \subseteq A,(*),(* *)$, and (***) imply that $\log |f(z)|=\int \log |f| d \mu$ for each $f \in R(K)^{-1}$. Thus $\mu$ is the logmodular measure for the complex homomorphism $z$ of $R(K)$, i.e., $\mu=\lambda_{z}$. But then (****) becomes $\phi(f)=\int f d \lambda_{z}$ for each $f \in A$. Since $\phi$ is multiplicative on $A, \lambda_{z}$ is now multiplicative on $A$. Hence Theorem B implies that $A=H^{\infty}\left(\lambda_{z}\right)$.

In [D, IV.1] it is shown that each measure in $M_{z}$ is mutually absolutely continuous with respect to $\lambda_{z}$. Thus in Theorem A, $\lambda_{z}$ can be replaced by any representing measure on $\partial K$ for the complex homomorphism $z$ of $R(K)$.

\section{BIBLIOGRAPHY}

[AS1] P. R. Ahern and D. Sarason, The $H^{p}$ spaces of a class of function algebras, Acta Math. 117 (1967), 123-163.

[AS2] On some hypodirichlet algebras of analytic functions, Amer. J. Math. 89 (1967), 932-941.

[D] J. J. Dudziak, The minimal normal extension problem for subnormal operators, J. Funct. Anal. 65 (1986), 315-338.

[Gam] T. W. Gamelin, Uniform algebras, Prentice-Hall, Englewood Cliffs, N. J., 1969.

[Gar] J. Garnett, On a theorem of Mergelyan, Pacific J. Math. 26 (1968), 461-467.

[S] E. L. Stout, The theory of uniform algebras, Bogden and Quigley, Tarrytown-on-Hudson, N. Y., 1971.

Department of Mathematics, Bucknell University, Lewisburg, Pennsylvania 17837 